\title{
ViBRIO WARNING
}

○ n March 13, 1990 the Health Department was notified of the death of a 53-year-old woman from septicaemia at a large Sydney teaching hospital. The woman had suffered from diabetes and chronic liver disease and was taking immunosuppressive drugs. Blood cultures taken before her death grew Vibrio vulnificus.

The patient's husband reported that he and his wife had consumed oysters on March 10 and 11. As V. vulnificus was also detected in samples of oysters taken from the same oyster lease in Brisbane Water, the woman's septicaemia was attributed to eating oysters. Three other cases of $V$. vulnificus primary septicaemia attributed to oyster consumption have been reported in NSW since 1988. All patients (males aged 64, 74, and 69 years) had chronic liver disease. One of these patients died.

Three cases of septicaemia following wound infection also occurred in the past 12 months in the same NSW north coast town where one of the men had lived. All three people had been wading in coastal rivers in the warmer months prior to presentation. No obvious wounds were seen on initial examination, but in all three, rashes occurred on the legs followed by cellulitis and septicaemia. V. vulnificus was grown from the wounds in each case. The first, a man in his 70 's on low doses of corticosteroids for asthma, died of septicaemia. The other two, a woman aged 55 who was treated with radiotherapy for cancer of the thyroid five years before, and a man of 65 with chronic airways disease, survived. Oyster consumption was not implicated in these cases.

These occurrences prompt the question as to whether people with chronic liver disease or other chronic conditions should be advised not to eat oysters.

\section{THE ORGANISM}

V. vulnificus is a member of the Vibrio family of bacteria. Vibrios are relatively salt tolerant (halophilic) gram negative rods which can ferment lactose. Eleven Vibrio species are pathogenic in man. Of these, the most important medically are V. cholera, V. parahaemolyticus and V. vulnificus'.

Halophilic Vibrios are natural inhabitants of estuary waters, occurring even in areas free from faecal contamination. Their survival depends on a number of environmental factors - most importantly water temperature. While the organism thrives in temperatures of $10-30^{\circ} \mathrm{C}$ it can survive at cooler temperatures when sediment is present. It favours salinity levels of 5-30\%, which are found in estuaries and inshore coastal areas. However, lower salinity levels may be tolerated where warmer temperatures and high levels of organic nutrient exist.

Cases of human infection with V. vulnificus have been reported in Japan, Belgium, the USA ${ }^{2}$ and Australia. Infection is rare. The incidence in Louisiana, USA, for example has been estimated to be 0.8 per 100,000 population. Infection is most likely to occur in summer and autumn.

The severity of infection appears to be related to both host and organism factors. An acidic polysaccharide capsule probably confers resistance to the bactericidal activity of human serum and to phagocytosis.
Virulent strains can use iron bound to transferrin (in haemoglobin and in haptoglobin complexes) for growth4.

The sensitivity of the organism to iron may help explain why iron overloading diseases are associated with a greater risk of severe disease. Cirrhosis may lead to increased levels of available iron, and deficiencies in neutrophil and macrophage function, or lead to bacterial leakage across the bowel wall or problems in clearing the organism from the enterohepatic circulation.

V. vulnificus produces a number of enzymes including cytotoxin-haemolysin, elastolytic protease, collagenase and phospholipases which may aid invasion of the body by the organism 1 .

\section{SEA FOOD}

Most pathogenic vibrios can maintain larger populations and live longer in environments with higher organisms ${ }^{1}$ such as plankton, fish and shellfish.

By filtering large amounts of water, shellfish can concentrate a variety of pathogenic aquatic organisms. Association with the flesh of filter feeding bivalve molluses after harvesting prolongs the life of pathogenic Vibrios outside their natural environments. The Vibrios can multiply if the molluses are stored in warm temperatures ${ }^{1}$. Pathogenic Vibrios can infect crustaceans, particularly in warmer climates, and to a lesser extent, fish. Studies in the USA have shown that over half of samples taken grew $V$. vulnificus during selected months. One study found that $11 \%$ of crabs sampled from a bay in Texas were infected ${ }^{5}$ in summer. A recent survey in NSW found V. vulnificus in about $40 \%$ of oysters sampled.

\section{CLINICAL MANIFESTATIONS}

V. vulnificus has been implicated in septicaemia and wound infections. In one study of 24 cases of primary septicaemia ${ }^{6}$, the illness typically began with chills, fever and prostration. One quarter had hypotension on admission, and three quarters developed secondary cutaneous lesions. The organism was cultured from the blood in $83 \%$ of cases.

Primary septicaemia is thought to occur after ingestion of the organism. V. vulnificus probably invades and replicates in the intestine causing inflammation and micro abscess formation. The organism produces toxins that destroy tissue and gains entry to the bloodstream through the portal vein or the intestinal lymphatic system ${ }^{1}$. The incubation period is short, with symptoms appearing about 16 hours after the organism is ingested. Overall more than half of patients with primary septicaemia die, and of those who develop hypotension within 12 hours of hospital admission, 90\% die.5.

In a review of three studies, Hoffmann et $\mathrm{al}^{7}$ found that 42 of 45 patients with primary septicaemia had underlying illnesses, and $76 \%$ had some type of liver disease. $53 \%$ of patients with primary septicaemia died.

A US case control study ${ }^{8}$ using all isolates of V. vulnificus received by the Centers for Diseases Control in 1981 and 1982 found that patients with primary $V$. vulnificus septicaemia were more likely to report having eaten raw oysters and have a history of liver disease, while patients 
with wound infection were more likely to have had recent exposure to salt water or shellfish.

Another multi-state US study ${ }^{6}$ identified ingestion of raw oysters as a risk factor for developing $V$. vulnificus septicaemia, but not for wound infection. Primary septicaemia occurred almost exclusively in people who had some underlying illness, particularly liver disease. Wound infection, on the other hand, resulted from exposure to seawater or freshly caught saltwater crabs, and appeared to affect people with no underlying illness. A case control study conducted in New Orleans ${ }^{3}$ found similar risk factors.

Liver disease, haemopoietic disorders, chronic renal insufficiency, haemochromatosis ${ }^{6}$, heavy alcohol intake and the use of immunosuppressants are risk factors for developing $V$. vulnificus septicaemia ${ }^{3}$. Other reported risk factors include thalassaemia ${ }^{4}$, diabetes, and leukaemia.

V. vulnificus wound infections may be mild and self limiting, or can progress to severe rapidly progressive cellulitis and myositis. The incubation period is short, with symptoms developing about 12 hours after contamination. The infection occurs in healthy as well as debilitated people 1 . Almost half (48\%) of cases in one study ${ }^{7}$ had an underlying disease such as diabetes, cirrhosis, gastric carcinoma and steroid-dependent asthma. While the presence of underlying disease does not appear to influence the risk of developing V. vulnificus wound infection, use of immunosuppressive drugs and having underlying diseases are associated with more severe outcomes for those who develop the condition.

V. vulnificus has also been reported in association with diarrhoea in patients reporting heavy alcohol intake, meningitis in a child with thalassaemia ${ }^{4}$, pulmonary infection, and endometriosis following undersea intercourse $^{1}$

\section{PREVENTION AND CONTROL}

Various recommendations have been made to reduce the risk of developing $V$. vulnificus infection, including:

Advising people with underlying illness to avoid shellfish injuries and contaminating any wounds with sea water during the warmer months ${ }^{1,7}$.

\section{INFLUENZA MONITORING}

here has been widespread community concern

that an influenza epidemic will spread within Australia this winter.

During the recent 1989-90 influenza epidemic [subtype A/England/427/88 (H3N2) - close to A/Shanghai/11/87 (H3N2)] in the United Kingdom, there was a reported peak number of some 800 deaths attributable to influenza ${ }^{1}$, the majority of these occurring in people over the age of 65 .

Worse influenza epidemics have occurred in the UK in the past. In 1969-70 an epidemic of A/Hong Kong/1/68 (H3N2) influenza occurred, with an estimated peak of 3,000 deaths per week, and in a 1975-76 A/Victoria/3/75 (H3N2) epidemic less than half as many influenza deaths were reported.

However, there are in fact important differences in the epidemiology of influenza between Australia and the UK. As can be seen from Fig 3, the UK had not experienced an epidemic of Influenza A (H3N2) since 1976. In contrast, Australians have been exposed to Influenza A (H3N2) virus in 1982, 1983, 1985 and 1989, making them less susceptible to these virus strains and decreasing the likelihood of a large influenza epidemic this year.

Should Australia experience an influenza epidemic in 1990 , initial cases could have occurred as early as May (as was the experience in the recent UK outbreak), or as late as July (as occurred in Australia during the moderate epidemic of 1989). The typical epidemic lasts five to nine weeks, peaking around the third to fifth week.

\section{Ongoing surveillance of viral isolates by}

Commonwealth Communicable Diseases Intelligence (CCDI) has revealed only 12 influenza isolates in the March and April periods - and only one was Influenza A subtype H3N2. It was noted that this was normal Influenza A activity for that particular time of year².

Compared with immunisation, natural acquisition of influenza appears to afford greater immunity against the disease and provides some protection against later emerging strains resulting from "antigenic drift'. This is the basis for not recommending influenza vaccine in otherwise healthy children and young adults.

To monitor the occurrence of influenza in the community, the Epidemiology and Health Services Evaluation Branch of the Department of Health and the Department of Community Medicine of the University of Sydney have established an influenza monitoring network. A total of 24 general practitioners throughout metropolitan Sydney will report on patients presenting with "'flu-like illness". These data will be collated with data from hospital separations and virology laboratories in order to identify the beginning, peak and end of an epidemic, if it does occur. 


\section{NEW MONOGRAPH ON HEART DISEASE}

A

monograph has just been published

by Department of Health, NSW, to help

community health staff promote better heart

health. The document, "Public screening for risk of heart disease", which is principally authored by Dr Karen Webb, provides guidelines and procedures for screening for cardiovascular risk factors. It can be obtained from the Health Promotion Unit, Department of Health, NSW, Level 23, McKell Bldg, Rawson Place, Sydney - phone (02) 2176666.

\section{TRAVEL HEALTH ADVICE}

ccess is now available for all Public Health Units to the Medical Advisory Service for Travellers Abroad (MASTA). The MASTA system is an information data bank on health and disease risks for overseas travellers which was developed at the London School of Hygiene and Tropical Medicine. It has been modified to Australian standards through the Tropical Health Program of the University of Queensland. The database, which is updated daily, provides the most up-to-date information on travel health currently available.

The Department of Health encourages all Area/Regional staff concerned with preventing infectious disease in travellers to discuss access to this database with Directors of Public Health Units. Further information can be obtained by contacting Mr Gavin Stewart on (02) 2176160.

\section{PUBLIC HEALTH EDITORIAL STAFF}

The Bulletin's editorial advisory panel is as follows: Dr Sue Morey, Chief Health Officer, Department of Health; Professor Stephen Leeder, Professor of Community Medicine, University of Sydney; Professor Geoffrey Berry, Professor of Epidemiology \& Biostatistics, University of Sydney; Dr Robert Reznik, Acting Director, Department of Community Medicine, Royal Prince Alfred Hospital; Professor Ian Webster, Professor of Community Medicine, University of NSW; Dr Christine Bennett, Acting Associate Director, Service Development, Department of Health; DrMichael Frommer, Epidemiologist, Epidemiology \& Health Services Evaluation Branch; Ms Jane Hall, Research Officer, Department of Community Medicine, Westmead Hospital; and Mr Michael Ward, Manager, Health Promotions Unit, Department of Health. The editor is Dr George Rubin, Director, Epidemiology and Health Services Evaluation Branch, Department of Health, NSW.

Design and Production - Health Public Affairs Unit, Department of Health, NSW.

The next issue of the Bulletin will include a letters section in addition to the articles, infectious disease notifications and news and comment sections.

Please send your articles, news, comments or letters to Dr George Rubin - P.O. Box K110 Haymarket NSW 2000 or $\operatorname{Fax}(02) 2175602$.

Suggestions for improving the reporting of infectious diseases are most welcome.

\section{FOUNDATION FELLOWSHIP}

T

he Australian Faculty of Public Health

Medicine was established by the RACP at its

May 1990 Annual General Meeting. Medical practitioners registered in Australia who have been predominantly engaged at any time in the practice of public health medicine may be eligible for Foundation Fellowship of the new Faculty.

Persons wishing to be considered for such Fellowship, should write for further information to:-

The Honorary Secretary

Australian Faculty of Public Health Medicine

145 Macquarie Street

SYDNEY NSW 2000

Phone: 2474461

Fax: 2313120

\section{Vibrio Warning}

\section{Continued from page 13}

\section{EDITORIAL NOTE}

The Department of Health recently consulted with senior Sydney physicians on appropriate recommendations for patients with chronic diseases. It was agreed that the risk of contracting V. vulnificus infection in NSW is very small. However, patients with chronic hepatic or renal disease, or those on immunosuppressive therapy should be advised to avoid eating oysters during the warmer months particularly after periods of heavy rain.

The Epidemiology and Health Services Evaluation Branch of the Department plans to initialc laboratory-based surveillance of isolations of $V$. vulnificus next spring.

Jeremy McAnulty MB BS, Registrar in Public Health Medicine, Epidemiology \& Health Services Evaluation Branch, Department of Health, NSW.

\section{References}

1. West PA, The human pathogenic Vibrios - a public health update with environmental perspectives. Epidem Inf, 1989;103:1-34. 2. Johnston JM, Andes WA, Glasser G, Vibrio vulnificus. A gastronomic hazard. JAMA, 1983;249:1756-57.

3. Johnston JM, Becker SF, McFarland LM, Vibrio vulnificus, man and the sea. JAMA, 1985;253:2850-2853.

4. Katz BZ, Vibrio vulnificus meningitis in a boy with thalassemia after eating raw oysters. Pediatrics, 1988;82:784-786.

5. Morris JG, Vibrio vulnificus - a new monster from the deep? Ann Int Med, 1988;109:261-263.

6. Blake PA, Merson MH, Weaver RE, Hollis DG and Heublein PC,

Disease caused by marine vibrio. $N$ Engl $J$ Med, 1979;300:1-5.

7. Hoffmann TJ et al, Vibrin vulnificus septicaemia. Arch Int Med, 1988;148:1825-1827.

8. Tacket CO, Brenner F, Blake PA, Clinical features and an epidemiological study of Vibrio vulnificus infections. I Infect Dis, 1984;149:558-561. 